\title{
Le galactosyl céramide : un nouveau récepteur pour le virus de l'immunodéficience humaine (VIH)
}

Le virus de l'immunodéficience humaine (VIH) infecte des lymphocytes et des macrophages exprimant à leur surface une glycoprotéine de $55 \mathrm{kDa}, \mathrm{CD} 4$. Cependant, le VIH est également capable d'infecter d'autres cibles cellulaires qui n'expriment pas cette molécule. Un deuxième récepteur pour le VIH vient d'être identifié dans deux types cellulaires différents, les oligodendrocytes du cerveau et les cellules épithéliales de la muqueuse intestinale. Il s'agit d'un glycosphingolipide, le galactosyl céramide. Le VIH interagit avec ce glycolipide par l'intermédiaire de la glycoprotéine externe de son enveloppe, la gp120, également impliquée dans la reconnaissance de la molécule CD4. L'infection des cellules $\mathrm{GalCer}^{+} / \mathrm{CD}^{-}$est efficacement inhibée par des anticorps monoclonaux anti-GalCer. Cette découverte pourrait permettre de développer de nouvelles approches pour lutter notamment contre la transmission et la pathogenèse du VIH au niveau de la muqueuse intestinale.

ADRESSES

J. Fantini: maître de conférences à l'université d'Aix-Marseille 1. Inserm U. 270, faculté de médecine secteur Nord, 13916 Marseille Cedex 20, France. N. Yahi : chercheur postdoctoral. Laboratoire de virologie, CHU de la Timone, 13005 Marseille, France.

$\mathrm{m} / \mathrm{s} \pi^{\circ} 8-9$ vol. 9, août-septembre 93 e syndrome d'immunodéficience acquise (SIDA) se caractérise par la disparition progressive des lymphocytes $T$ auxiliaires chez les individus infectés par le VIH. Ces lymphocytes portent à leur surface une glycoprotéine de $55 \mathrm{kDa}, \mathrm{CD} 4$, qui s'est révélée un récepteur de très haute affinité pour la glycoprotéine de surface de l'enveloppe du VIH, la gp120 [1]. CD4 est également impliquée dans l'infection des monocytes et des macrophages, qui jouent un rôle important dans la propagation du virus in vivo. L'infection des lymphocytes et des macrophages peut être bloquée in vitro par des formes solubles de la molécule CD4, et par certains anticorps monoclonaux (Leu3A, OKT4A) reconnaissant le site de fixation de la gp120 localisé dans la région amino-terminale de CD4 [2]. Le VIH pénètre dans la cellule hôte par un mécanisme de fusion entre l'enveloppe du virus et la membrane plasmique de la cellule cible. 


\section{RÉFÉRENCES}

1. Klatzmann D, Champagne E, Chamaret $\mathrm{S}$, et al. T lymphocyte $\mathrm{T} 4$ molecule behaves as the receptor for human retrovirus LAV. Nature $1984 ; 312$ : 767-8.

2. Sattentau $Q$, Dalgleish AG, Weiss RA, Beverley P. Epitopes of the CD4 antigen and HIV infection. Science $1986 ; 234$ 1120-3.

3. Maddon PJ, Dalgleish AG, McDougal JS, Clapham R, Weiss RA, Axel R. The T4 gene encodes the AIDS virus receptor and is expressed in the immune system and the brain. Cell 1986 ; 47 : 333-48.

4. Chesebro B, Buller R, Portis J, Wehrly K. Failure of human immunodeficiency virus entry and infection in CI4-positive human brain and skin cells. J Virol 1990 ; $64: 215-21$.

5. Clapham PR, Blanc D, Weiss RA. Specific cell surface requirements for the infection of CD4-positive cells by human immunodeficiency virus types 1 and 2 and by simian immunodeficiency virus. Virology $1991 ; 181: 703-15$.

6. Dragic T, Charneau P, Clavel F. Alizon M. Complementation of murinc cells for human immunodeficiency virus envelope/CD4-mediated fusion in human/murine hetcrokaryons. J Virol $1992 ; 66: 4794-802$.

7. Hildreth JEK, Orentas RJ. Involvement of a leukocyte adhesion receptor (LFA-1) in HIV-induced syncitium formation. Science $1989 ; 244$ : 1075-8.

8. Ashorn PA, Berger EA, Moss B. Human immunodeficiency virus envelope glycoprotein CD4-mediated fusion of non primate cells with human cells. J Virol $1990 ; 64$ : 2149-56.

9. Hattori T, Koito A, Takatsuki K, Kido $\mathrm{H}$, Katunuma $\mathrm{N}$. Involvement of tryptaserelated protease(s) in human immunodeficiency type 1 infection. FEBS Lett 1989 ; 248 : 48-52.

10. Clements GJ, Price-Jones MJ, Stephens PE, et al. The V3 loop of the HIV-1 and HIV-2 surface glycoproteins contain protcolytic cleavage sites : a possible function in viral fusion? AIDS Res Hum Retrovir $1991 ; 7: 3-16$.

11. Stephens PE, Clements G, Yarranton GT, Moore J. A chink in HIV's armour. Nature $1990 ; 343$ : 219

12. Fauci AS. The human immunodeficiency virus : infectivity and mechanisms of pathogenesis. Science 1988 ; 239 : 617-22.

13. Embretson J, Zupancic M, Beneke J, et al. Analysis of human immunodeficiency virus-infected tissues by amplification and in situ hybridization reveals latent and permissive infections at single-cell resolution. Proc

\section{Rôle de CD4} dans l'infection par le VIH

Cette étape du cycle d'infection du virus est encore mal comprise et plusieurs arguments permettent de penser que des facteurs cellulaires pourraient être indispensables à l'entrée du virus après l'étape de fixation. En effet, le VIH est incapable d'entrer dans une cellule murine transfectée avec l'ADN complémentaire de l'ARNm codant pour CD4. En fait, ces cellules exprimant un CD4 humain fixent le VIH avec autant d'affinité que des cellules humaines, mais la fusion virus-cellule n'a pas lieu [3]. Parmi les cellules humaines $\mathrm{CD}^{-}{ }^{-}$, certaines (lignée HeLa) deviennent sensibles à l'infection après introduction d'un vecteur d'expression de CD4. Cependant, dans certains cas (lignées U87MG et SCL1), l'expression induite de CD4 permet la fixation du VIH, mais pas son entrée $[4,5]$. L'ensemble de ces résultats suggère qu'un facteur spécifique présent dans certaines cellules humaines est nécessaire à l'entrée du virus, sa fixation sur le récepteur CD4 n'étant pas suffisante pour qu'il y ait internalisation. L'équipe du Dr Marc Alizon (Paris, ICGM, Inserm U. 332) a récemment confirmé cette hypothèse [6]. Ces chercheurs ont mis au point un test, fondé sur la transactivation du gène de la $\beta$ galactosidase bactérienne (lac $Z$ ) placé sous le contrôle de la séquence LTR du VIH-1, qui permet de visualiser par histochimie la fusion de cellules murines exprimant CD4 avec des cellules exprimant l'enveloppe du VIH-1, indépendamment de la réplication du virus. Grâce à ce test, ils ont montré que des hétérocaryons (cellules murines-CD4+/cellules humaines) pouvaient fusionner avec les cellules exprimant l'enveloppe du virus, tandis que des hétérocaryons (cellules murines-CD4+/cellules simiennes) restaient résistantes à la fusion. Le facteur d'origine humaine nécessaire à la fusion virus-cellule, après fixation de la gp120 au CD4, n'est pas identifié à l'heure actuelle. Plusieurs co-récepteurs possibles ont été envisagés par différentes équipes. L'intégrine LFA-1, qui joue un rôle important dans la formation des syncitiums induits par le VIH [7], est un candidat potentiel. Néanmoins, cette intégrine n'est pas exprimée par les cellules HeLa-CD4, qui sont pourtant sensibles à l'infection par le VIH [8]. Plus récemment, deux groupes ont suggéré l'implication dans le mécanisme de fusion d'une protéase de surface, chargée de cliver la gp120 au niveau de la boucle V3 [9, 10]. En effet, en dépit de sa grande variabilité, la boucle V3 semble conserver un site de clivage pour des protéases de type trypsine ou chymotrypsine [10]. La coupure protéolytique de la gp120 recombinante au niveau de ce site a été observée dans des cellules de hamster $\mathrm{CHO}$ [11]. Cependant, l'expression de CD4 à la surface de ces cellules ne les rend pas sensibles à l'infection [5]. Le rôle exact du clivage de la boucle de V3 de la gp120 reste donc à déterminer.

Bien entendu, ces travaux ne remettent pas en cause l'importance de CD4 dans l'infection par le VIH. In vivo, les cellules infectées par le VIH sont principalement des lymphocytes et des macrophages exprimant CD4 [12]. Cependant, en combinant les techniques de détection par PCR in situ et hybridation in situ, Embretson et al. [13] ont récemment démontré l'existence de cellules d'adénocarcinomes, $\mathrm{CD}^{-}{ }^{-}$, infectées par le VIH in vivo. Ces résultats sont en accord avec certaines publications faisant état d'un mode d'entrée du virus, indépendant de CD4, dans des lignées de fibroblastes [14], de cellules nerveuses [15] et de cellules épithéliales de côlon [16, 17]. L'expression de CD4 par ces cellules n'a pas pu être mise en évidence, et leur infection n'est inhibée ni par le CD4 soluble ni par les anticorps monoclonaux anti-CD4 (Leu3A, OKT4A) qui bloquent l'infection CD4 dépendante des lymphocytes T. Bien que les cellules infectées dans ces expériences soient exclusivement des cellules de lignées, il était intéressant de rechercher, à l'aide de ces modèles, le ou les récepteurs éventuellement responsables de l'attachement du virus.

\section{Le galactosyl céramide, récepteur du VIH dans les cellules nerveuses}

A la suite de ces observations, $\mathrm{m} / \mathrm{s} n^{\circ} 8-9$ vol. 9, août-seplembre 93 
l'équipe du Dr Gonzalez-Scarano (Philadelphie, PA, USA) a entrepris une étude systématique de l'infection de lignées de cellules nerveuses CD4- par le VIH. En utilisant une panoplie d'anticorps monoclonaux dirigés contre des molécules exprimées à la surface de ces cellules, ces chercheurs ont pu caractériser l'une d'entre elles, le galactosyl céramide (GalCer), comme un récepteur potentiel du VIH dans le système nerveux [18]. Cette molécule, dont la structure chimique est rappelée sur la figure 1, est un marqueur des oligodendrocytes et des cellules de Schwann. L'équipe de Philadelphie a montré que des anticorps monoclonaux et polyclonaux anti-GalCer empêchaient l'entrée du VIH dans les cellules nerveuses en inhibant la fixation de la gp120 au glycolipide. Ces expériences ont permis d'identifier pour la première fois un récepteur du VIH différent de la molécule CD4.

\section{Le GalCer, récepteur intestinal du VIH}

La muqueuse intestinale joue un rôle fondamental dans la transmission et la pathogenèse du VIH. L'épithélium colo-rectal représente très certainement une voie prépondérante d'infection lors de rapports sexuels par voie anale $[19,20]$. En outre, différents auteurs ont clairement établi la présence d'ARN messagers et de protéines du VIH dans les différents types cellulaires de l'épithélium intestinal et colo-rectal chez l'individu infecté : cellules épithéliales de la crypte [21, 22], cellules entérochromaffines [23], cellules absorbantes [24, 25], cellules à mucus [24]. L'infection de ces cellules par le VIH pourrait alors être à l'origine des désordres entéropathiques (malabsorption, diarrhées, amaigrissement) chez les malades atteints de SIDA [26-28]. Il est donc essentiel de connaître les mécanismes impliqués dans l'entrée du VIH dans les cellules de l'épithélium intestinal. La lignée d'adénocarcinome de côlon humain HT29 s'est avérée être un modèle de choix pour aborder cette étude $\left(\mathrm{m} / \mathrm{s} n^{\circ} 2\right.$, vol. 8, p. 178). En effet, les cellules HT29 peuvent être infectées par de nombreux isolats (souches de référence ou isolats pri$\mathrm{m} / \mathrm{s} n^{\circ} 8-9$ vol. 9, août-seplembre 93

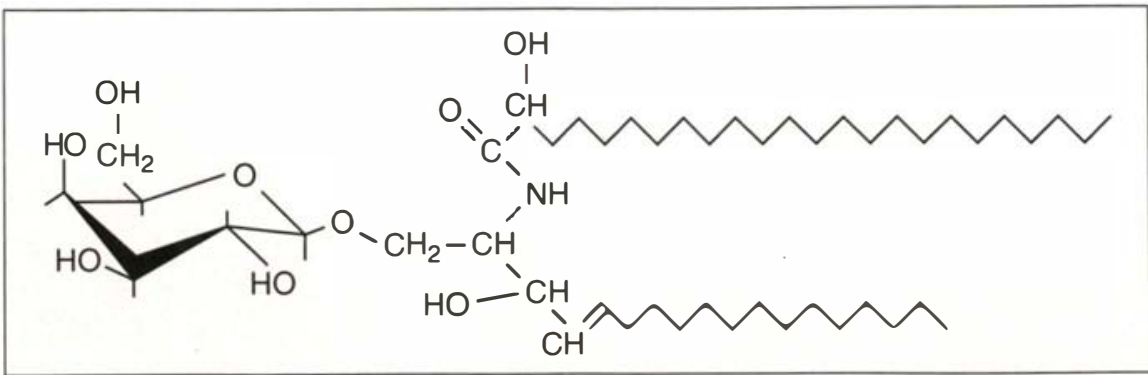

Figure 1. Structure du galactosyl céramide (GalCer).

maires) du VIH, et dans certains cas (VIH-1 [IIIB], VIH-1 [NDK], VIH-2 [MEN]), le virus peut se répliquer activement dans ces cellules. Trois jours après l'infection, on détecte une activité transcriptase inverse, témoin de la réplication du virus, dans les surnageants de culture des cellules. Cette activité transcriptase inverse augmente progressivement au fur à mesure des passages successifs pour atteindre une valeur plateau environ trois mois après l'infection dans le cas du virus VIH-1 $\left(\mathrm{NDK}^{*}\right)$. Une évolution comparable est observée par dosage de l'antigène viral p24gag pour les virus VIH-1 (IIIB) et VIH-1 (NDK), la production maximale du virus se stabilisant aux alentours de $150 \mathrm{ng}$ de $\mathrm{p} 24 \mathrm{gag} / \mathrm{ml}$ de surnageant de culture/24 heures. Le titre infectieux du virus produit par les cellules HT29 est alors de $10^{5} \mathrm{TCID}^{50} / \mathrm{ml}$ (test d'infectivité sur cellules lymphocytaires MT4 et CEM). L'augmentation de l'activité transcriptase inverse et de la concentration de l'antigène $p 24^{\text {gag }}$ rend probablement compte d'une amplification de l'infection due aux virus produits par les cellules HT29. Le pourcentage de cellules infectées a pu être déterminé par immunofluorescence indirecte à l'aide d'un anticorps monoclonal anti-p24ag (figure 2). Une semaine après l'infection, une faible proportion de cellules (inférieure à $5 \%$ de la population globale) est marquée avec cet anticorps (figure $2 A)$. Cette proportion augmente progressivement pour atteindre environ $20 \%$ un mois après l'infection (figure $2 B$ ). Elle se stabilise à $50 \%$ des cellules à partir de trois mois après l'infection et au-delà (figure 2C).

* NDK : nom d'un isolat du VIH, au même titre que $L A V, I I I B$, etc.
Les données de la microscopie électronique et le fait que l'infection des cellules HT29 soit insensible aux agents lysosomotropes, tels que chloroquine ou $\mathrm{NH}_{4} \mathrm{Cl}$, permettent d'éliminer la possibilité d'un mécanisme d'endocytose pour l'entrée du VIH dans ces cellules. Le virus doit donc pénétrer à l'intérieur des cellules HT29 par un mécanisme de fusion après avoir interagi avec un récepteur membranaire. Ce récepteur est différent de $\mathrm{CD} 4$, puisque l'infection des cellules HT29 n'est inhibée par aucun anticorps monoclonal antiCD4 [16, 17]. En outre, après traitement de la surface cellulaire par de fortes doses de trypsine (qui clive la molécule CD4 et abolit la sensibilité des lymphocytes $\mathrm{T}$ à l'infection), les cellules HT29 sont toujours infectables par le VIH (N. Yahi et J. Fantini, manuscrit en préparation). Ces résultats nous ont incités à rechercher la présence du GalCer à la surface des cellules HT29 [29]. Le marquage immunocytochimique présenté dans la figure 3 montre qu'environ $50 \%$ des cellules HT29 expriment un antigène de surface reconnu par un anticorps monoclonal anti-GalCer. Ce pourcentage correspond à la proportion de cellules infectées par le VIH Afin de confirmer la nature du glycolipide reconnu par cet anticorps, nous avons analysé des extraits lipidiques de cellules HT29 par chromatographie sur couche mince [30]. Il apparaît que le GalCer est un glycolipide abondamment représenté dans les cellules HT29 (figure 4, ligne B2). Parmi les glycolipides présents dans les cellules HT29, il est le seul à être reconnu par la gp 120 du $\mathrm{VIH}$, dans un test de fixation sur plaque de chromatographie (figure 4, ligne A2). L'anticorps monoclonal anti-GalCer que nous avons utilisé inhibe l'infec- 


\section{RÉFÉRENCES}

14. 'Tateno M, Gonzalez-Scarano F. I,evy JA. Human immunodeficiency virus can infect CD4-negative human fibroblastoid cells. Proc Natl Acad Sci USA 1989; 86 : 4287-90.

15. Harouse JM, Kunsch C, Hartle H'I, et al. CD4-independent infection of human neural cells by human immunodeficiency virus type 1. J Virol $1989 ; 63: 2527-33$

16. Omary MB, Brenner DA, de Grandpré I,Y, Roebuck KA, Richman IDI) Kagnoff MF. HIV-1 infection and expression in human colonic cells : infection and expression in $\mathrm{CD}^{+}{ }^{+}$and $\mathrm{CD}^{-}$- cell lines. AIIDS $1991 ; 5$ : 275-81.

17. Phillips DM, Bourinbaiar AS. Mechanism of HIV spread from lymphocytes to epithclia. Virology1992; 186: 261-73

18. Harouse JM, Bhat S, Spitalnik SI, et al. Inhibition of entry of HIV-1 in neura cell lines by antibodies against galactosyl ceramidc. Science 1991; 253 : 320-3.

19. Ischner T, Hussain L, Wilson J, Chapman M. Mucosal transmission of HIV. Nature 1991; 353 : 709

20. Sabin AB. Improbability of effective vaccination against human immunodeficiency virus because of its intracellular transmission and rectal portal of entry. Proc Natl Acad Sci USA 1992 ; 89 : 8852-5.

21. Kotler DP, Reka S. Borcich A. Cronin WJ. Detection, localization, and quantitation of HIV-associated antigens in intestinal biopsies from patients with HIV. $A m$ Pathol 1991; 139 : 823-30.

22. Mathijs JM, Hing $M$, Grierson $J$, Dwyer IDE, Goldschmidt C, Cooper DA Cunningham AL. HIV infection of rectal mucosa. Lancet 1988; 1 : 1111

23. Nelson JA. Wiley CA, Reynolds-Kohler C, Reese CE, Margaretten W, Levy JA. Human immunodeficiency virus detected in bowel epithelium from patients with gastrointestinal symptoms. Lancet 1988; 1 : 259-62.

24. I evy JA, Margaretten W, Nelson J Detection of HIV in enterochromaflin cells in the rectal mucosa of an AIDS patient. Ann J Gastroenterol $1989 ; 84$ : 787-9.

25. Heise C, Dandekar S, Kumar P Duplantier R, Donovan R, Halsted CH. Human immunodeficiency virus infection of enterocytes and mononuclear cells in human je junal mucosa. Gastroenterology $1991 ; 100$ : 1521-7.

26. Kotler DP, Gaetz HP, Klein EB, Lange M, Holt PR. Enteropathy associated with the acquired immunodeficiency virus. $A n n$
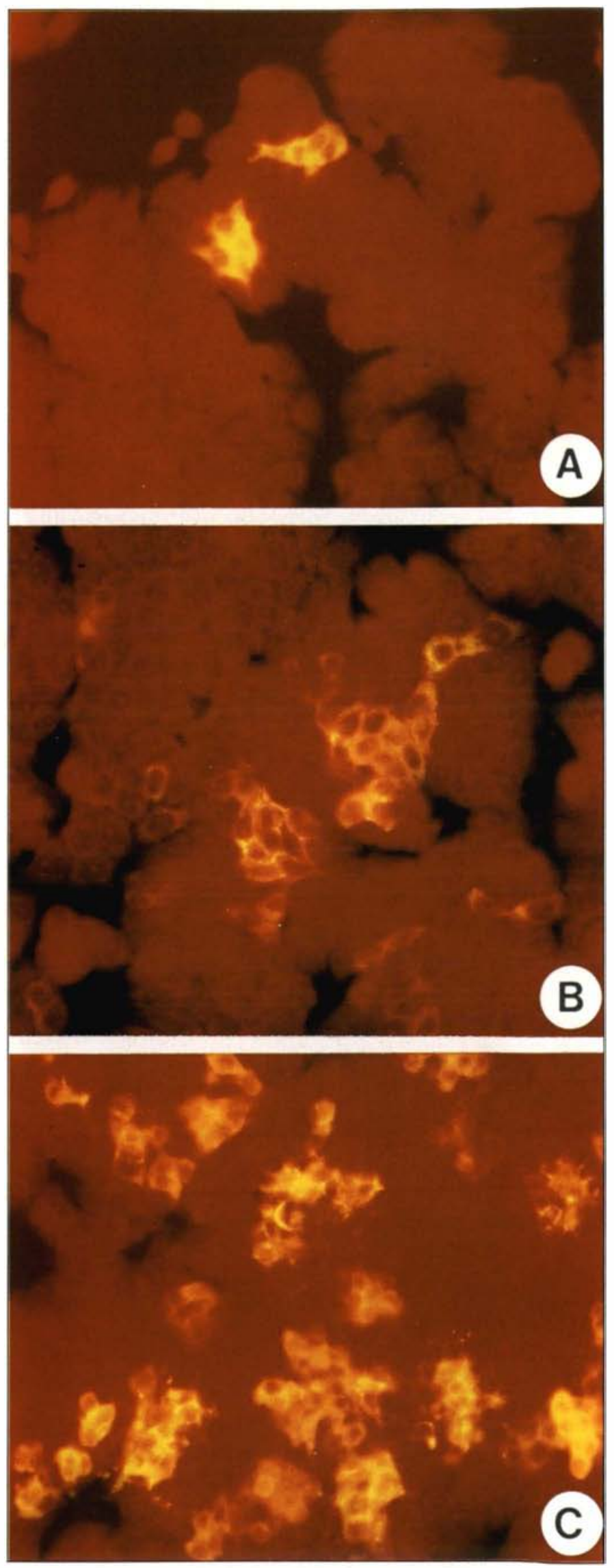

$m / s n^{\circ} 8-9$ vol. 9, août-septembre 93 

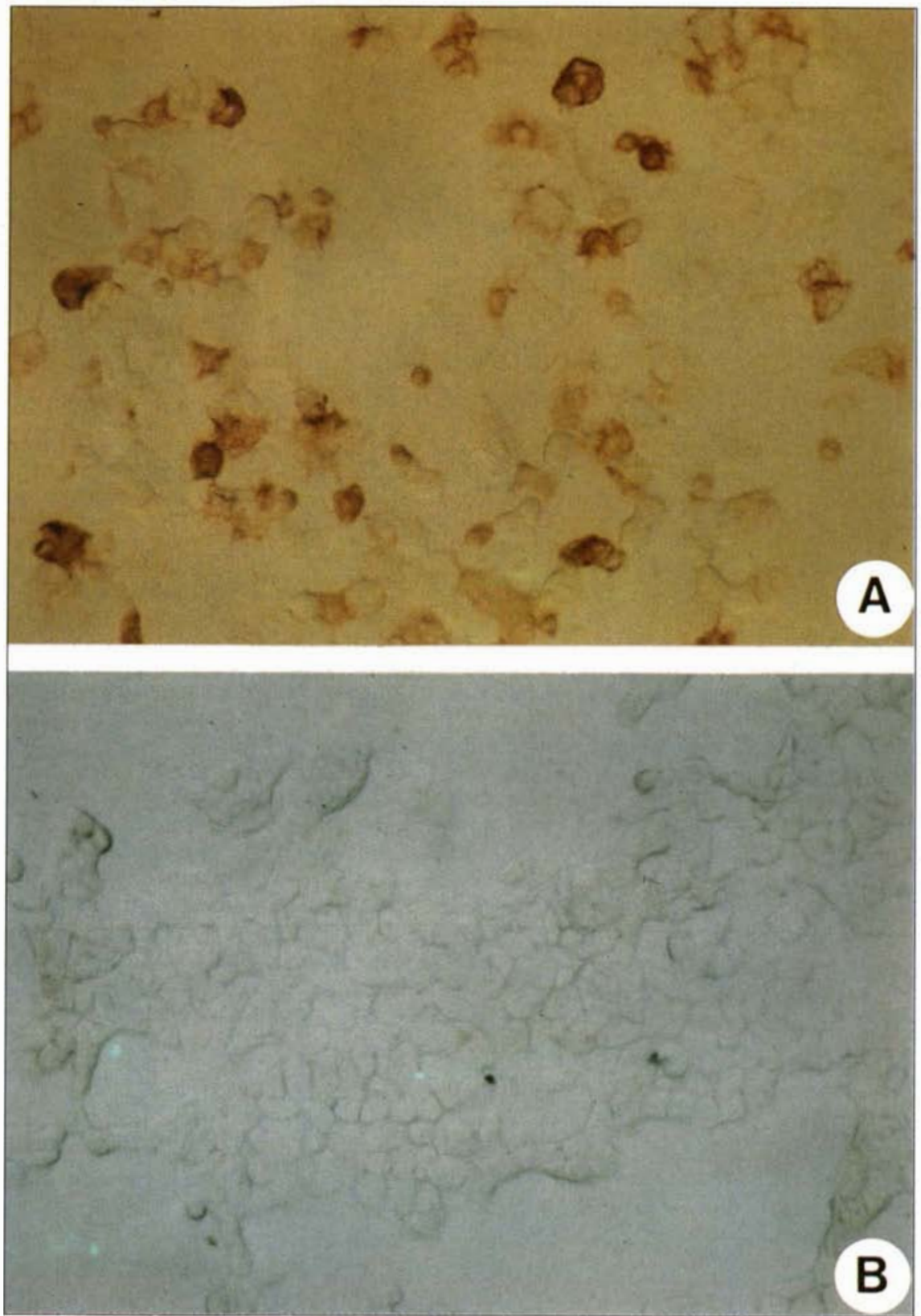

Figure 3. Mise en évidence du GalCer à la surface des cellules HT29. L'anticorps monoclonal anti-GalCer (une IgG3 de souris) [40] reconnaît environ $50 \%$ des cellules HT29 (A). La fraction IgG3 d'un sérum de souris, utilisée comme contrôle de l'expérience d'immunocytochimie (marquage à la peroxydasel, ne réagit pas avec les cellules HT29 (B).

Figure 2. Détection de l'antigène viral p24gag dans les cellules HT29 infectées. Les cellules HT29 infectées par l'isolat $\mathrm{VIH}-1$ (NDK) sont fixées dans un mélange éthanol/acide acétique une semaine (A), un mois (B) ou deux ans (C) après l'infection. Le marquage fluorescent obtenu à l'aide d'un anticorps monoclonal spécifique de l'antigène viral p24gag permet de visualiser les cellules infectées en jaune/vert dans la population uniformément colorée en rouge par le bleu d'Evans [30].

$\mathrm{m} / \mathrm{s} n^{\circ} 8-9$ vol. 9, aoút-septembre 93 tion des cellules HT29 [29, 30] en bloquant l'internalisation du VIH (figure 5).

La démonstration formelle qu'une molécule cellulaire donnée joue le rôle de récepteur pour un virus nécessite un certain nombre d'arguments expérimentaux permettant de satisfaire à au moins trois critères : (1) la mise en évidence d'une interaction physique entre le virus (ou l'un de ses constituants) et cette molécule ; (2) le fait que des anticorps, dirigés contre le récepteur potentiel, soient capables de bloquer la fixation et/ou l'entrée du virus dans la cellule cible ; (3) la présence du récepteur doit être une condition sine qua non de la fixation du virus à la cellule cible et donc de l'infection ; à l'inverse, l'absence du récepteur doit être associée à la résistance des cellules à l'infection.

Nous avons montré que le GalCer remplissait les deux premières conditions : interaction GalCer-gp120 et blocage de l'infection par des anticorps anti-GalCer. Pour satisfaire au troisième critère, deux approches expérimentales étaient a priori envisageables: (1) induire l'expression du GalCer dans une cellule non infectable afin de la rendre sensible à l'infection par le VIH ; (2) obtenir des clones dérivés de lignées hétérogènes pour l'expression du GalCer, afin d'établir une corrélation entre cette expression et la sensibilité de chacun de ces clones à l'infection par le VIH. La première approche était délicate car elle nécessitait la réactivation d'une voie métabolique complexe. Nous avons donc opté pour la seconde, c'est-à-dire le clonage de deux lignées intestinales, HT29 et Caco-2 [30]. Il ressort de cette étude que tous les clones exprimant le GalCer sont infectables par plusieurs isolats du VIH-1, alors que les clones qui ne l'expriment pas ne sont pas infectables. La présence du GalCer apparaît donc nécessaire à l'infection des cellules épithéliales du côlon in vitro. D'autres auteurs ont établi que le GalCer était le glycolipide principal des cellules épithéliales de la muqueuse de l'intestin grêle et du côlon humains [31]. Les résultats obtenus in vitro à l'aide de lignées n'étant pas toujours extrapolables in vivo, nous avons entrepris la purification du GalCer à partir de l'épithé- 


\section{RÉFÉRENCES}

27. Ullrich $R$, Zeitz $M$, Heise $M$, L'age M, Hoffken G, Rieken Eo. Small intestinal structure and function in patients infected with human immunodeficiency virus (HIV). Evidence for HIV-induced enteropathy. Ann Intern Med 1989 ; 111 : 15-21.

28. Yolken RH, Li S, Perman J, Viscidi R. Persistent diarrhea and fecal sheldding of retroviral nucleic acids in children infected with human immunodeficiency virus. $J$ Infect Dis 1991; $164: 61-6$.

29. Yahi N, Bagdiguian S, Moreau $\mathrm{H}$, Fantini J. Galactosyl ceramide (or a closely related molecule) is the receptor of human immunodeficiency virus type 1 on human colon epithelial HT29 cells. J Virol 1992 ; $66: 4848-54$

30. Fantini J, Cook DG, Nathanson N, Spitalnik SL, Gonzalez-Scarano F. Infection of colonic epithelial cell lines by type 1 human immunodeficiency virus (HIV-1) is associated with cell surface expression of galactosyl ceramide, a potential alternative gp120 receptor. Proc Natl Acad Sci USA $1993 ; 90: 2700-4$.

31. Holgersson J, Jovall PA, Breimer ME. Glycosphingolipids of human large intestine : detailed structural characterization with special reference to blood group compounds and bacterial receptor structures. $J$ Biochem 1991 ; 110 : 120-31.

32. Rousset $\mathbf{M}$. The human colon carcinoma cell lines HT29 and Caco-2 : two in vitro models for the study of intestinal differentiation. Biochimie 1986; 68 : 1035-40.

33. Svensson L, Finlay BB, Bass D, von Bonsdorff $\mathrm{CH}$, Grenberg HB. Symmetric infection of rotavirus on polarized human intestinal epithelial (Caco-2) cells. J Virol $1991 ; 65: 4190-7$.

34. Karlsson KA. Animal glycosphingolipids as membrane attachment sites for bacteria. Annu Rev Biochem 1989 ; 58 : 309-50.

35. Bhat S, Spitalnik SL, Gonzalez-Scarano F, Silberberg DH. Galactosyl ceramide or a derivative is an essential component of the neural receptor for human immunodeficiency virus type 1 envelope glycoprotein gp120. Proc. Natl Acad Sci USA 1991; 88 : 7131-4.

36. Bartlett JG, Belitsos PC, Sears CL. AIDS enteropathy. Clin Infect Dis $1992 ; 15$ : 726-35.

37. Ullrich $\mathrm{R}$, Heise $\mathrm{W}$, Bergs $\mathrm{C}$, L'age M, Riecken EO, Zeitz M. Effects of zidovudin treatment on the small intestinal mucosa in patients infected with the human immunodeficiency virus. Gastroenterology $1992 ; 102$ : 1483-92.

38. Bélec L, Trotot P, Lescs MC, Gray F. Lésions précoces du système nerveux central au cours de l'infection par le virus de l'immunodéficience humaine. médecine/scien-

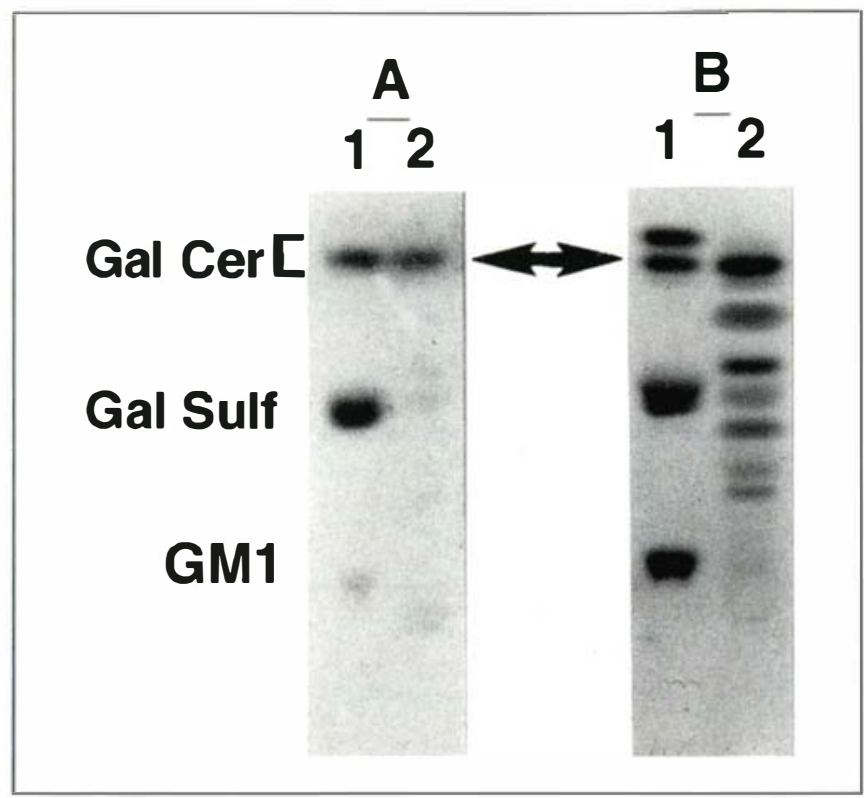

Figure 4. Analyse des glycolipides des cellules HT29 par chromatographie sur couche mince. (A) Fixation de la gp120. Après chromatographie, les plaques sont successivement incubées avec la gp 120 puis des anticorps de lapin anti-gp120 et enfin des anticorps de chèvre anti-IgG de lapin radioiodés. Le document présenté est une photographie de l'autoradiogramme obtenu. (B) Visualisation des glycolipides par coloration à l'orcinol. Ligne 1 : glycolipides standard: galactosyl céramide (GalCer), galactosyl sulfatide (GalSulf) et ganglioside GM1 de cerveau de bœuf. Noter les deux formes (hydroxylée ou non) du GalCer du cerveau de bœuf (ligne B1). Seule la forme hydroxylée (marquée d'une flèche) est reconnue par la gp120 (ligne A1). Ligne 2 : glycolipides neutres des cellules HT 29 obtenus après extraction de Folch (phase inférieure). La gp 120 reconnait exclusivement le GalCer parmi ces glycolipides (ligne A2). (Les détails techniques relatifs à ces expériences sont décrits dans [30].)

lium intestinal d'individus non infectés et nous avons ainsi pu montrer qu'il était capable, comme le GalCer des cellules HT29, de fixer la gp120 [30]. Nous pouvons donc conclure que le GalCer permet très probablement la fixation du VIH à la surface des cellules de l'épithélium intestinal.

\section{Métabolisme du GalCer}

Le GalCer appartient au groupe des glycosphingolipides, qui sont les principaux glycolipides des tissus animaux. On les trouve essentiellement dans le feuillet externe de la membrane plasmique où ils contribuent abondamment aux motifs glucidiques des surfaces cellulaires. Les glycosphingolipides sont constitués de l'association d'une molécule de sphingosine, d'un acide gras et d'un ou plusieurs oses (figure 6). L'acide gras, de longueur variable (16 à 26 atomes de carbone), est lié à la sphingosine par une liaison amide pour constituer le céramide. Le céramide correspond à la partie hydrophobe du glycosphingolipide, ancrée dans la membrane plasmique. Le cérébroside est défini comme étant une céramide monohexoside, l'hexose étant lié au C1 du céramide par une liaison $\beta$ glycosidique. Cet hexose est soit du D-glucose (glucocérébroside ou glucosyl céramide, GluGer), soit du Dgalactose (galactocérébroside ou galactosyl céramide, GalCer). Le glucose et le galactose sont dans une configuration $\beta$, ce qui implique que la liaison entre le sucre et le céramide est de type $\beta 1$-glycosidique. Le GalCer peut être converti en sulfogalactosyl céramide (galactosyl sulfatide, 


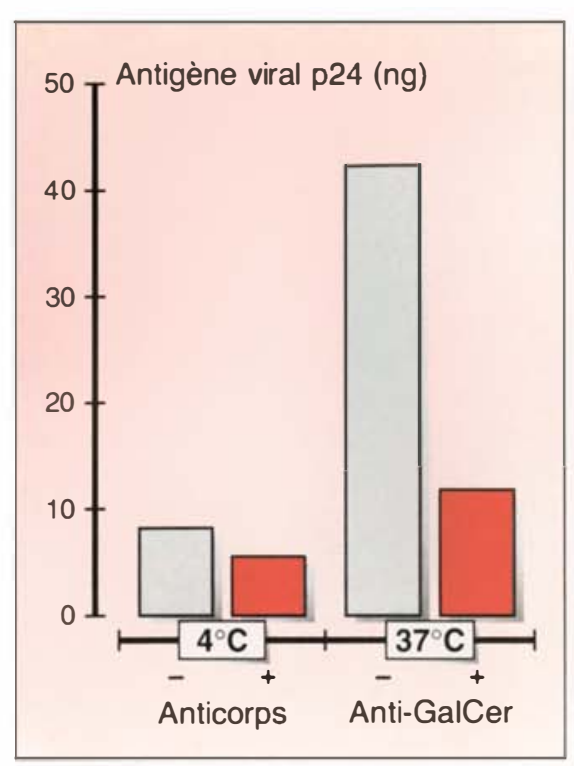

Figure 5. L'anticorps monoclonal anti-GalCer bloque l'internalisation du VIH-1HT29. Les cellules HT29 sont prétraitées à $4{ }^{\circ} \mathrm{C}$ pendant 1 heure par les anticorps anti-GalCer $(+)$, ou par des IgG3 de souris (-), puis sont exposées au $\mathrm{VIH}-1$ (isolat NDK) pendant 1 heure toujours à $4^{\circ} \mathrm{C}$. Une partie des cultures est alors maintenue à $4{ }^{\circ} \mathrm{C}$ tandis qu'une autre est placée à $37^{\circ} \mathrm{C}$ pendant 1 heure. L'internalisation du virus n'ayant lieu qu'à $37^{\circ} \mathrm{C}$, l'expérience à $4{ }^{\circ} \mathrm{C}$ est donc une expérience de contrôle. Les cellules sont ensuite lavées et traitées par de la trypsine pour détruire les particules virales non internalisées. La présence du virus à l'intérieur des cellules est mesurée par dosage de l'antigène p24gag après solubilisation des cellules.

GalSulf) présent en grande quantité dans la myéline. Les voies métaboliques de synthèse de ces composés sont schématisées sur la figure 7 .

La sphingosine est amidifiée par un acylcoenzyme A (acylCoA) formant un céramide, précurseur des autres sphingolipides. La fonction alcool primaire d'un céramide peut être substituée par des oses activés pour former les deux types de cérébrosides, puis des sulfatides ou des gangliosides. La biosynthèse du GalCer nécessite un transfert du groupement sucré de l'UDP-galactose sur une molécule de céramide (figure 6). Cette réaction est catalysée par une galactosyl transférase spécifique dont la $\mathrm{m} / \mathrm{s} n^{\circ} 8-9$ vol. 9, coût-septembre 93

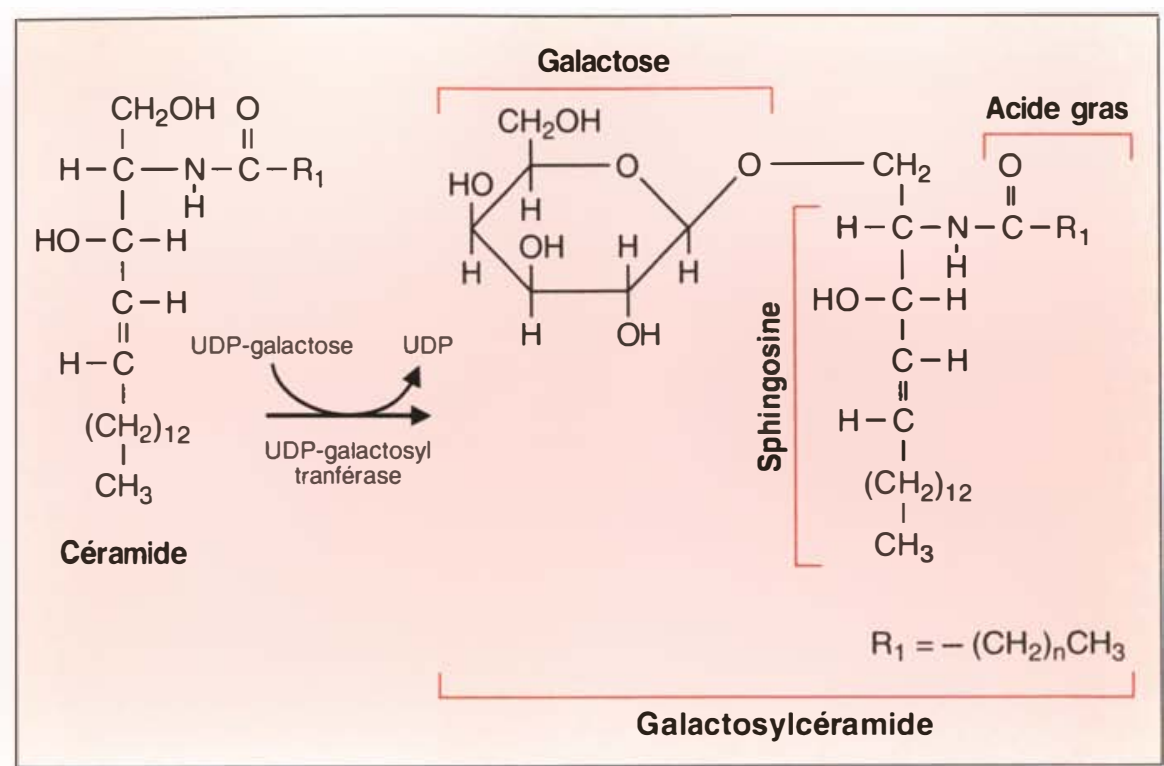

Figure 6. Biosynthèse du GalCer.

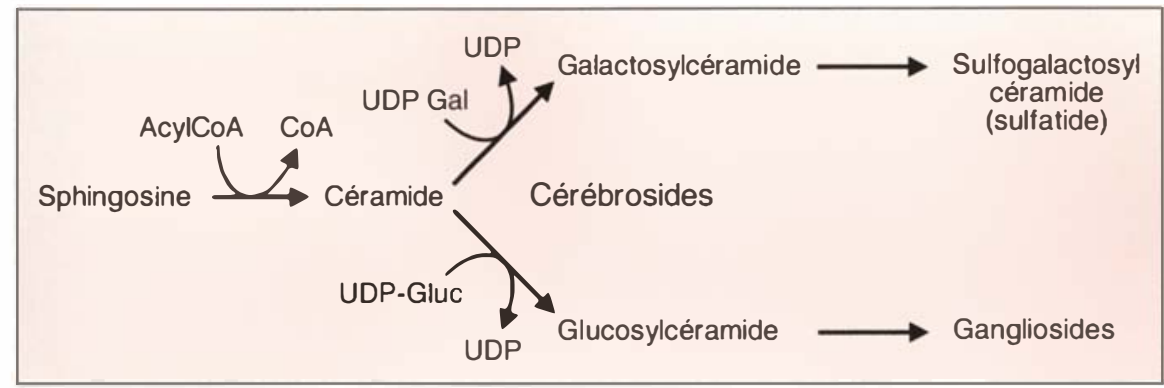

Figure 7. Voies métaboliques de synthèse des sulfatides et des gangliosides.

présence est indispensable pour la formation de GalCer dans une cellule.

\section{Polarité de l'infection des cellules épithéliales}

La principale caractéristique d'une cellule épithéliale est la séparation de sa membrane plasmique en deux domaines distincts : la membrane apicale, constituée de microvillosités, interagit avec le milieu extérieur (la lumière intestinale par exemple), et la membrane basolatérale fait face au milieu intérieur. Ces deux domaines, séparés par des jonctions de type étroit, ont une ultrastructure et une composition biochimique spécifiques qui permettent aux cellules épithéliales d'exercer des fonctions polarisées. Les cellules HT29 (ou des clones dérivés de ces cellules) acquièrent in vitro une différenciation de ce type lorsqu'on les cultive dans un milieu carencé en glucose [32]. Les cellules forment alors une monocouche épithéliale qui représente un modèle intéressant de l'épithélium intestinal. Le VIH est capable d'infecter les deux faces de cette monocouche polarisée $\left(\mathrm{m} / \mathrm{s} n^{\circ} 2\right.$, vol. 8, p. 178). La répartition ubiquitaire du GalCer dans les domaines apical et basolatéral de la membrane plasmique des cellules HT29 différenciées permet de comprendre pourquoi l'infection n'est 


\section{RÉFÉRENCES}

39. Bansal R, Pfeiffer SE. Reversible inhibition of oligodendrocyte progenitor differentiation by a monoclonal antibody against surface galactolipids. Proc Natl Acad Sci USA $1989 ; 86: 6181-5$

40. Ranscht B, Clapshaw PA, Price J, Noble M, Seifert W. Development of oligodendrocytes and Schwann cells studied with a monoclonal antibody against galactocerebroside. Proc Natl Acad Sci USA 1982 ; $79: 2936-47$

41. Dyer CA, Benjamins JA. Galactocerebroside and sulfatide independently mediate $\mathrm{Ca} 2+$ responses in oligodendrocytes. I Neurosci Res 1991; 30 : 699-711.

42. Macher BA, Swecley CC. Glycosphingolipids : structure, biological source and properties. Meth Enzymol 1978 ; 50 : 236-50.

43. Lingwood CA, Quinn PA, Wilansky S, Nutikka A, Ruhnke HL, Miller RB. Common sulfoglycolipid receptor for mycoplasma involved in animal and human infertility. Biol Reprod 1990 ; 43 : 694-7.

44. Kubushiro K, Mikami M, Nozawa S, Ishizuka R, Iwamori M, Nagai Y. Menstrual cycle-associated alteration of sulfogalactosylceramide in human uterine endometrium : possible induction of glycolipid sulfation by sex steroid hormones. Arch Biochem Biophys $1989 ; 268$ : 129-36.

45. Krivan HC, Olson LD, Barile MF, Ginsburg V, Roberts DD. Adhesion of Mycoplasma pneumoniae to sulfated glycolipids and inhibition by dextran sulfate. $J$ Biol Chem 1989 ; 264 : 9283-8.

46. Monco JC, Villar BF, Rogers RC, Szczepanski A, Whecler CM, Benach JL. Borrelia burgdorferi and other related spirochetes bind to galactocerebroside. Neurology $1992 ; 42$ : 1341-8.

47. Paruchuri DK, Seifert HS, Ajioka RS, Karlsson KA. Identification and characterization of a Neisseria gonorthoeae gene encoding a glycolipid binding adhesin. Proc Natl Acad Sci USA 1990 ; 87: 333-7.

48. Kielczynsci W, Harrison I,C, I,cedman $\mathrm{PJ}$. Direct evidence that ganglioside is an integral component of the thyrotropin receptor. Proc Natl Acad Sci USA 1991; 88 : 1991-5.

49. Brennan MJ, Hannah JH, I ceininger E. Adhesion of Bordetella pertussis to sulfatides and to the GalNac $\beta 4 \mathrm{Gal}$ sequence found in glycosphingolipids. J Biol Chem 1991; 226 : 18827-31.

50. Srnka CA. Tiemeyer M, Gilbert JH, et al. Cell surface ligands for rotavirus : mouse intestinal glycolipids and synthetic carbohy- pas polarisée [29]. Une observation analogue a récemment été décrite par Svensson et al. [33] : la souche SA11 $\mathrm{du}$ rotavirus, qui interagit avec le ganglioside GA1 (ou asialo-GM1), est capable d'infecter les deux pôles des cellules Caco-2. Ce récepteur glycolipidique est donc très probablement présent dans les deux domaines membranaires de ces cellules polarisées. Mais ce cas n'est pas général. Beaucoup de virus infectant des cellules épithéliales se fixent sur un récepteur dont l'expression est restreinte à l'un des pôles de la monocouche (SV40, parvovirus, virus de la stomatite vésiculaire, virus de la forêt de Semliki, virus de l'influenza).

\section{Bases structurales de l'interaction GalCer-gp 120}

Ces dernières années, de nombreux glycolipides membranaires ont été caractérisés en tant que sites récepteurs pour divers agents pathogènes [34], principalement des bactéries et des virus (Tableau I). Certains glycolipides interagissent aussi avec des toxines bactériennes ou même des hormones. Le GalCer et son dérivé sulfaté, le galactosyl sulfatide (GalSulf), sont eux-mêmes responsables de la fixation de mycoplasmes (Mycoplasma hominis, M. pneumoniae) et de bactéries (Borrelia burgdorferi, Treponema phagedenis). La reconnaissance de motifs de nature glycolipidique par des ligands et des organismes aussi variés n'est pas surprenante, si l'on considère que les glycolipides membranaires constituent une part importante des glycoconjugués de la membrane plasmique, aux côtés des glycoprotéines et des protéoglycanes. La surface d'une cellule est recouverte d'une très grande variété de motifs glucidiques qui représentent autant de structures potentielles pour l'interaction avec les agents pathogènes. La composition membranaire en glycolipides varie d'un tissu à l'autre, ce qui rend bien compte de la spécificité de l'infection pour certains types cellulaires. Ainsi, les cellules épithéliales de l'intestin sont riches en lactosyl céramide (LacCer), un glycosphingolipide de type Gal-GlcCer qui est reconnu par de nom- breuses bactéries telles que Neisseria gonorrhoeae, l'agent causal de la blennoragic (Tableau I). Il est intéressant de constater que la gp120 du VIH-1 n'est pas capable de reconnaître ce récepteur. Cette propriété est d'autant plus remarquable que certains anticorps monoclonaux antiGalCer se fixent indifféremment au GalCer et au LacCer (J. Fantini, résultats non publiés). L'interaction GalCer-gp120 revêt donc des caractéristiques bien spécifiques que l'on peut analyser à l'aide de molécules dérivées du GalCer dans un test de fixation de la gp120 sur des plaques de chromatographie sur couche mince [35]. La chaîne d'acide gras ne semble pas importante pour l'interaction puisque la gp120 se fixc de manière similaire sur des molécules de GalCer contenant du palmitate, du stéarate ou du nervonate. En revanche, la présence du galactose est indispensable, puisque l'interaction n'a pas lieu lorsque le sucre est un résidu de glucose (GluCer) ou en absence de sucre (céramide). La substitution du groupement $\mathrm{OH}$ sur l'atome de carbone $\mathrm{n}^{\circ} 3 \mathrm{du}$ galactose (groupement 3'OH) par un groupement sulfate (GalSulf) ou un acide neuraminique (ganglioside GM4) n'a pas d'effet sur l'interaction. On peut donc conclure que la gp120 reconnaît la liaison $\beta 1$-glycosidique entre la galactose et le céramide, ainsi qu'une partie de la molécule de galactose ne comprenant pas le groupement 3'OH du sucre. La position du groupement 4'OH, qui détermine la différence entre les deux épimères glucose et galactose, est bien entendu essentielle pour l'interaction. En outre, les cellules de l'épithélium intestinal sont riches en glycolipides de forme hydroxylée. L'hydroxylation de la chaîne d'acide gras des glycosyl céramides détermine la conformation et l'orientation de la partie glycanique au niveau de la membrane plasmique [34]. En ce qui concerne le GalCer, seule la forme hydroxylée (celle que l'on trouve dans les cellules HT29) est capable de reconnaître la gp120 du VIH-1 (figure 4). Cela suggère que la forme non hydroxyléc possède une conformation qui n'est pas compatible avec l'interaction du glycolipide avec la glycoprotéine virale. 
Tableau I

EXEMPLES DE GLYCOLIPIDES RÉCEPTEURS D'AGENTS PATHOGĖNES, DE TOXINES OU D'HORMONES

\begin{tabular}{|c|c|c|c|}
\hline Glycolipide & Structure & Agent pathogène ou ligand & Référence \\
\hline Galactosyl céramide & Gal $\beta 1-$ Cer & $\begin{array}{l}\text { Mycoplasma hominis } \\
\text { Mycoplasma pneumoniae } \\
\text { Treponema phagedenis } \\
\text { Borrelia burgdorferi } \\
\text { gp120 du VIH }\end{array}$ & $\begin{array}{l}{[43]} \\
{[45]} \\
{[46]} \\
{[46]} \\
{[18]}\end{array}$ \\
\hline Lactosyl céramide & Gal $\beta 1-4 G l c \beta 1-C e r$ & $\begin{array}{l}\text { Neisseria gonorrhoeae } \\
\text { Campylobacter jejunii } \\
\text { Shigella dysenteriae } \\
\text { Clostridium botulinum } \\
\text { Candida albicans }\end{array}$ & $\begin{array}{l}{[47]} \\
{[34]} \\
{[34]} \\
{[34]} \\
{[34]}\end{array}$ \\
\hline GM1 & $\begin{array}{c}\text { Gal } \beta 1 \text {-3GalNac } \beta 1-4 \text { Gal } \beta 1-4 \mathrm{Glc} \beta 1-\text { Cer } \\
\text { NeuAc } \alpha 2-3\end{array}$ & $\begin{array}{l}\text { Thyrotropine } \\
\text { Toxine cholérique }\end{array}$ & $\begin{array}{l}{[48]} \\
{[34]}\end{array}$ \\
\hline Asialo GM1 & Gal $\beta 1-3 G$ alNac $\beta 1-4 \mathrm{Gal} \beta 1-4 \mathrm{Galc} \beta 1-\mathrm{Cer}$ & $\begin{array}{l}\text { Bordetella pertussis } \\
\text { Rotavirus }\end{array}$ & $\begin{array}{l}{[49]} \\
{[50]}\end{array}$ \\
\hline
\end{tabular}

\section{Rôles possibles du GalCer dans la pathogenèse du VIH}

Un dysfonctionnement gastrointestinal est fréquemment observé chez les individus infectés par le VIH [26, 27]. La diarrhéc constitue le symptôme le plus fréquemment rencontré, survenant dans 30 à $80 \%$ des cas au cours de l'infection. Elle cst parfois associée à des douleurs abdominales, à de la fièvre ou à une perte de poids, qui est l'un des critères du stade évolutif de l'ARC* qui précède le SIDA. Cette diarrhéc peut aussi s'accompagner d'une malabsorption intestinale qui se traduit par une réduction des taux sanguins d'albumine, d'acide folique, de vitamine B12, de calcium, de zinc et parfois par une anémic qui peut être chronique. Sur le plan biochimique, cette malabsorption se manifeste par un déficit de certaines enzymes de la membrane apicale des entérocytes. L'origine de ces dérèglements est certainement complexe [36]. Ils peuvent être causés par des infections opportunistes : champignons (Candida albicans), virus (cytomégalovirus, virus de l'herpès), bactéries (Mycobacterium

\footnotetext{
* ARC : AIDS (acquired immune deficiency syndrome) related complex.

avium) ou protozoaires (Cryptosporidium). Mais souvent, il est impossible de détecter, dans l'intestin des patients, un agent infectieux autre que le VIH pouvant rendre compte de ces dysfonctionnements. Ce constat a conduit certains autcurs [27] à postuler que le virus lui-même pouvait être à l'origine de ce syndrome entéropathique (entéropathie induite par le VIH). Cette hypothèse a été confortée par la mise en évidence d'ARN et de protéines du virus dans certaines cellules de l'épithélium intestinal in vivo [21-25]. Par ailleurs, l'ARN viral est souvent détecté dans les fèces d'enfants infectés par le VIH lorsqu'ils sont atteints du syndrome de diarrhée peristante [28]. Il est donc envisageable que la réplication du VIH dans la muqueuse intestinale soit en partie responsable du défaut de maturation entérocytaire. En effet, dans les cellules HT29 infectées in vitro, la production de particules virales est associée à d'importantes modifications ultrastructurales : hypertrophie de l'appareil de Golgi, accumulation de grains de sécrétion, différenciation abortive de la membrane apicale $\left(m / s n^{\circ} 2\right.$, vol. 8, p. 178). Chez les patients traités par l'AZT, on constate généralement une amélioration des fonctions d'absorption due à une augmentation des activités enzy- matiques des entérocytes (lactase/ $\beta$ glucosidase, phosphatase alcaline) [37].

Les atteintes du système nerveux central sont également très fréquentes au cours de l'infection par le VIH. Il est communément admis que le virus est introduit dans les tissus cérébraux par l'intermédiaire de macrophages infectés qui franchissent la barric̀re hémato-encéphalique. La présence du VIH dans le système nerveux central est associée à de nombreuses lésions : encéphalite, méningite, troubles neuropsychologiques. Les causes de ces lésions sont là encore très complexes. Des réactions immunopathologiques provoquées par le VIH y participent probablement [38].

L'identification d'un récepteur pour la gp120 du VIH-1 dans le système nerveux central et l'épithélium intestinal permet de formuler de nouvelles hypothèses pour expliquer les désordres causés localement par le virus. Plusieurs équipes ont montré l'importance du GalCer (ou de son dérivé GalSulf) dans les mécanismes accompagnant la différenciation des oligodendrocytes. Cultivées en présence d'anticorps anti-GalCer, ces cellules sont incapables de se différencier et restent bloquées à un stade précoce de leur développement tant que les anticorps ne sont pas reti- 
rés [39]. Des expériences similaires, réalisées sur des cellules de Schwann, ont montré l'importance du GalCer et du GalSulf dans la formation de la gaine de myéline dont dépend la croissance de l'axone [40]. Les anticorps anti-GalCer agissent en induisant un influx de calcium, qui va entraîner à son tour une dépolymérisation des microtubules [41]. La conséquence de cette stimulation est un phénomène de dé-différenciation cellulaire, qui présente des analogies avec la démyélinisation des cellules nerveuses chez les patients infectés par le VIH. Dans le système nerveux central, la stimulation du GalCer par la gp120, libre ou associée à des particules virales, pourrait induire une réponse de ce type. Ce mécanisme peut aussi être envisagé dans les entérocytes exposés au VIH. La fixation de la gp120 sur le GalCer, en modifiant les concentrations intracellulaires de calcium, pourrait provoquer une dépolymérisation des éléments cytosquelettiques nécessaires à la maturation de la membrane apicale des entérocytes. Il faut insister sur le fait que les mécanismes évoqués ne nécessitent pas forcément l'infection des cellules, intestinales ou nerveuses, par le VIH. La production de gp120 par les macrophages dans le cerveau, ou par les lymphocytes et les macrophages dans la muqueuse intestinale, pourrait à elle seule perturber le fonctionnement des cellules GalCerpositives dans ces tissus [35]. L'étude de ces mécanismes est en cours dans notre laboratoire. La répartition tissulaire du GalCer et des glycolipides apparentés est encore mal connue. Pendant longtemps, on a pensé que ces structures étaient spécifiques du système nerveux central [42]. La démonstration de l'expression du GalCer dans la muqueuse intestinale et de sa capacité à fixer la gp120 du VIH-1 devrait stimuler de nouvelles recherches pour identifier les types cellulaires exprimant des glycolipides de ce type. A cet égard, des travaux récents faisant état de la présence du GalSulf dans les spermatozoïdes [43] et les cellules endométriales de l'utérus [44] sont compatibles avec le rôle potentiel de ces cellules dans la transmission hétérosexuelle du VIH [19, 20]. En ce qui concerne les lymphocytes $\mathrm{CD}^{+}{ }^{+}$, ils ne semblent pas exprimer de GalCer [29], ce qui permet a priori d'écarter un rôle potentiel de ce glycolipide comme corécepteur de CD4 dans ces cellules.

\section{Conclusion}

Les travaux consacrés au VIH ont montré que ce rétrovirus pouvait infecter des tissus variés en utilisant des récepteurs différents, dont deux au moins (CD4 et GalCer) ont été caractérisés. D'autres structures membranaires impliquées dans l'interaction du VIH avec les surfaces cellulaires seront peut-être identifiées prochainement. Il pourra s'agir de co-récepteurs liés à CD4 (intégrines de type LFA-1, protéases assurant le clivage de la boucle V 3 de la gp120, glycolipides ou protéoglycanes) et nécessaires à la fusion virus-cellule, ou bien de récepteurs à part entière permettant la fixation du virus sur un type cellulaire donné. Nous pourrons alors peut-être développer de nouvelles stratégies anti virales qui, prenant en compte la nature des cellules cibles, permettront de limiter la dissémination du virus dans l'organisme et de prévenir les pathologies spécifiquement induites par le VIH dans chaque tissu infecté

\section{Remerciements}

Nous remercions très sincèrement H. Moreau, G. Rougon (Cnrs UR A 179) et $\mathrm{S}$. Baghdiguian (université de Montpellier 2) pour leur collaboration dans la réalisation de ce travail. Une partie du projet a été poursuivie à l'université de Pennsylvanie à Philadelphie en collaboration avec l'équipe de F. Gonzalez-Scarano que nous remercions tout particulièrement. Nous tenons également à exprimer notre gratitude envers S. Spitalnik, N. Nathanson, J. Harouse, K.I. Stefano, D. Kolson, J. Hoxie, A. Albright, D. Cook et C. Griot pour leurs critiques constructives. Nos travaux ont été financés par l'université d'Aix-Marseille 1, la Fondation pour la recherche médicale, l'Otan, l'Inserm et l'ANRS.

\section{Summary}

Galactosyl ceramide : a new receptor for the human immunodeficiency virus (HIV)

HIV infects lymphocytes and macrophages that express $\mathrm{CD} 4$, a $55 \mathrm{kDa}$ cell surface glycoprotein. However, the ability of HIV to infect CD4-negative cells suggests the existence of an alternate, non$\mathrm{CD} 4$ receptor for the virus. $\mathrm{Re}$ cently, a glycosphingolipid called galactocerebroside or galactosyl ceramide (GalCer) was proposed as a candidate $\mathrm{HIV}-1$ receptor in neural cells and in colon epithelial cells. Gp120, the external envelope glycoprotein of $\mathrm{HIV}-1$ that recognizes $\mathrm{CD} 4$, is involved in the interaction with GalCer. The viral glycoprotein binds also to the sulfated derivative of $\mathrm{GalCer}$, but totally ignores closely related molecules such as glucosyl and lactosyl ceramide. HIV infection of GalCer ${ }^{+} / \mathrm{CD}^{-}$- cells is efficiently inhibited by anti-GalCer monoclonal antibodies. These findings should be important in view of the development of new antiHIV strategies, especially for the prevention of HIV transmission and pathogenesis in the gastrointestinal mucosa.

\section{TIRÉS A PART}

\section{J. Fantini.}

